\title{
A New Record of Fruit Fly (Diptera: Tephritidae) From Turkey and Contributions to Fauna of Gaziantep Province
}

\author{
Mehmet Yaran ${ }^{1, a, *}$, Vedat Görmez ${ }^{2, b}$, Mürşit Koyuncu ${ }^{2, c}$ \\ ${ }^{I}$ Department of Medical Services and Techniques, Islahiye Vocational School, Gaziantep University, 27800 İslahiye/Gaziantep, Turkey \\ ${ }^{2}$ Department of Biology, Faculty of Science and Art, Gaziantep University, 27310 Gaziantep, Turkey
}

${ }^{*}$ Corresponding author

\begin{tabular}{l|l}
\hline A R T I C LE I N F O & A B S T R A C T \\
\hline Research Article & $\begin{array}{l}\text { Fruit flies (Diptera: Tephritidae) is one of the most important Diptera families, some species damage } \\
\text { fruits, and cause millions of dollars in every years. In this study, adult fruit fly materials were collected } \\
\text { from Gaziantep province between } 2010 \text { and } 2021 \text { from possible host plants using insect net. Obtained } \\
\text { materials were examined, and were determined that } 15 \text { species. All species recorded for the first time } \\
\text { from Gaziantep province. In addition, Goniurellia tridens (Hendel, 1910) recorded as a new record } \\
\text { from Turkey. With these contributions, Gaziantep fruit fly fauna increased to 44 species. In the article, } \\
\text { examined materials, current list of Gaziantep fruit fly fauna and adult and wing figures of Goniurellia } \\
\text { tridens (Hendel, 1910) were given. }\end{array}$ \\
Received : 22/06/2021
\end{tabular}

Keywords:

Tephritidae

Fruit flies

Fauna

New record

Gaziantep

Türk Tarım - Gıda Bilim ve Teknoloji Dergisi, 9(12): 2233-2237, 2021

\section{Türkiye'den Yeni Bir Meyve Sineği (Diptera: Tephritidae) Kaydı ve Gaziantep İli Faunasına Katkılar}

\begin{tabular}{l|l}
\hline M A K A L E B İ L G İ S I & Ö Z \\
\hline Araştırma Makalesi & $\begin{array}{l}\text { Meyve sinekleri familyası (Diptera: Tephritidae), en önemli sinek familyalarından birisidir, bazı türler } \\
\text { meyvelere zarar verir ve her y1l milyonlarca dolarlı zarara neden olur. Bu çalışmada, ergin meyve } \\
\text { sineği örnekleri Gaziantep ilinden, böcek atrabi kullanılarak muhtemel konukçu bitkiler üzerinden } \\
\text { 2010 ve 2021 y1lları arasinda toplanmıştır. Elde edilen örnekler incelenmiş ve 15 tür belirlenmiştir. } \\
\text { Kaydedilen tüm türler Gaziantep ilinden ilk kez tespit edilmiştir. Ek olarak, Goniurellia tridens } \\
\text { (Hendel, 1910) türü Türkiyeden il kez bu çalıma ile kaydedilmiştir. Bu katk1lar ile birlikte, Gaziantep } \\
\text { meyve sinekleri faunas1 44 türe yükselmiştir. Makalede, incelenen materyal, Gaziantep meyve } \\
\text { sinekleri faunasinın güncel listesi ve Goniurellia tridens (Hendel, 1910) türünün ergin ve kanat } \\
\text { fotoğrafları verilmiştir. }\end{array}$ \\
$\begin{array}{l}\text { Geliş }: 22 / 06 / 2021 \\
\text { Kabul }: 29 / 07 / 2021\end{array}$ \\
$\begin{array}{l}\text { Anahtar Kelimeler: } \\
\text { Tephritidae }\end{array}$
\end{tabular}

Tephritidae

Meyve sinekleri

Fauna

Yeni kayit

Gaziantep 


\section{Introduction}

Tephritidae is one of the largest families of Diptera order and it includes about 492 genera and, 4716 species (Pape et al., 2011). Several species of fruit flies (Diptera: Tephritidae) are invasive pests of horticultural crops worldwide, due to their adaptation to various regions, high polyphagia and rapid reproduction (Sarwar, 2015).

In recent years, many studies carried out to determine fruit flies fauna in Turkey. Several species recorded or identified as new over the two decades. In the recent studies, 9 new species (Terellia askaleensis Kütük et al., 2011; Tephritis ozaslani Kütük et al., 2012; Terellia freidbergi Korneyev et al., 2013; Urophora turkeyensis Yaran and Kütük, 2014; Heringina arezoana Namin and Korneyev, 2015, Carpomya liat Freidberg, 2016; Terellia akguli Yaran et al., 2018; Tephritis turkeri Kütük and Yaran, 2020; Tephritis kutuki Yaran and Görmez, 2020) and 14 new records (Acidia cognata (Wiedemann, 1817), Campiglossa sororcula (Wiedemann 1830), Carpomya wiedemanni (Meigen, 1826), Chetostoma curvinerve Rondani, 1856, Dacus ciliatus Loew, 1862, Hemilea dimidiata (Costa, 1844), Ictericoides zelleri (Loew, 1844), Tephritis zeryni Hendel, 1927, Terellia armeniaca Korneyev, 1985, Terellia ivannikovi Korneyev et al., 2013, Terellia nigronota Korneyev, 1985, Terellia plagiata (Dahlbom, 1850), Urophora tenuis Becker, 1908, Urophora trinervii Korneyev and White, 1996) have been reported from Turkey (Görmez, 2011; Bayrak, 2011; Kütük et al., 2011, 2012; Korneyev et al., 2013; Kütük et al., 2013; Yaran and Kütük, 2014, 2015, 2016; Korneyev and Kolcsar, 2015; Namin and Korneyev, 2015; Freidberg, 2016; Yaran et al., 2018a, 2018b; Çalışkan Keçe et al., 2019; Görmez and Kütük, 2020; Kütük and Yaran, 2020; Yaran and Görmez, 2020). Number of fruit fly species has increased significantly in recent years with new species, and records, and 173 species have known up to date in Turkey.

Up to date, 29 fruit fly species recorded in Gaziantep province of Turkey (Yaran, 2009; Yaran et al., 2018b). In the study, 15 fruit fly species were determined in different locations of Gaziantep, and Goniurellia tridens (Hendel, 1910) identified for the first time from Turkey. This paper provides locations of collected specimens, figures of new record, and current list of fruit flies in Gaziantep province.

\section{Materials and Methods}

According to previous studies (Yaran, 2009; Yaran et al., 2018b), 29 fruit fly species were reported in the fauna of Gaziantep province. In this study, new specimens were collected and identified between 2010 and 2021 years in order to contribute to the fauna of Gaziantep province. Obtained specimens were identified using following literatures and provided keys; Hendel (1927), White (1988), Freidberg and Kugler (1989), Korneyev and White (1993, 1999), Kütük (2003a, 2006), Korneyev (2003, 2006), Merz (1994), Görmez (2011), Korneyev et al. (2013) and Yaran (2014). All the specimens are stored in the Gaziantep University, Biology Department, Entomology Laboratory.

\section{Results and Discussions}

In this article, some contributions reported to the fruit fly fauna of Gaziantep province. Together with this contribution, number of fruit fly species increased to 44 species in Gaziantep province. In addition, Goniurellia tridens (Hendel, 1910) identified for the first time from Turkey, and number of fruit flies increased to 174.

Updated list of Tephritidae presented in Table 1 and, contributed species were given alphabetical order in the below. In addition, figures of male and female adults (wing, head and thorax) of new record presented in the below.

\section{Actinoptera discoidea (Fallen, 1814)}

Material examined: 1 q, Gaziantep, İslahiye, Fevzipaşa, $37^{\circ} 05^{\prime} \mathrm{N}, 37^{\circ} 37^{\prime} \mathrm{E}, 523 \mathrm{~m}, 10.05 .2021$; Det: Yaran M. (Leg. Yaran, Görmez and Koyuncu).

Bactrocera oleae (Rossi, 1790)

Material examined: $2 \hat{\partial}, 1$, Gaziantep, Şehitkamil, Bilek, $37^{\circ} 12^{\prime} \mathrm{N}, 37^{\circ} 56 \mathrm{E}, 513 \mathrm{~m}, 10.07 .2018$; Det: Yaran M. (Leg. Yaran, Görmez and Koyuncu).

Chaetorellia jaceae (Robineau - Desvoidy, 1830)

Material examined: 3 ๙ิ $\widehat{\jmath}, 2$ 우, Gaziantep, İslahiye,

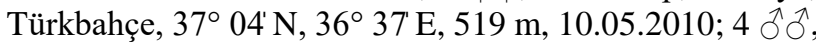
2 우, Şahinbey, Türközü, $36^{\circ} 05^{\prime} \mathrm{N}, 37^{\circ} 21^{\prime} \mathrm{E}, 691 \mathrm{~m}$, 10.05.2010; $2 \hat{\delta} \hat{\sigma}^{2}$, Center, $37^{\circ} 00^{\prime} \mathrm{N}, 37^{\circ} 19^{\prime} \mathrm{E}, 843 \mathrm{~m}$, 10.05.2010; 1 गे, Oğuzeli, $36^{\circ} 54^{\prime} \mathrm{N}, 37^{\circ} 32^{\prime} \mathrm{E}, 643 \mathrm{~m}$, 03.06.2010; 1 ô, Karkamış, $36^{\circ} 49^{\prime} \mathrm{N}, 37^{\circ} 45^{\prime} \mathrm{E}, 507 \mathrm{~m}$, 03.06.2010; 1 ${ }^{\lambda}, 1$ ㅇ, Araban, Yukar1 Karavaiz, $37^{\circ} 27^{\prime} \mathrm{N}$, $37^{\circ} 47^{\prime} \mathrm{E}, 554 \mathrm{~m}, 16.06 .2010 ; 1$ ㅇ, Araban, Suvarlı, $37^{\circ} 33^{\prime}$

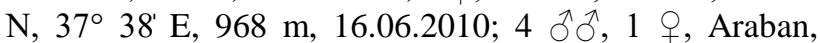

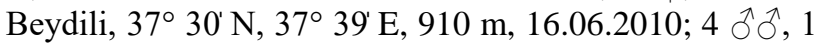
q, Şahinbey, Kürüm, $36^{\circ} 57^{\prime} \mathrm{N}, 37^{\circ} 09^{\prime} \mathrm{E}, 903 \mathrm{~m}$, 17.06.2010; 1 ๙⿱ , Şahinbey, Uğurtepe, $36^{\circ} 57^{\prime} \mathrm{N}, 37^{\circ} 05^{\prime} \mathrm{E}$, 774m, 17.06.2010; 1 त, Şahinbey, Cebeler, $37^{\circ} 02^{\prime} \mathrm{N}, 37^{\circ}$ 16 E, 942 m, 01.07.2010; Det: Yaran M. (Leg. Yaran, Görmez and Koyuncu).

Euleia heraclei (Linnaeus, 1758)

Material examined: 4 을 Gaziantep, Araban, $36^{\circ} 53^{\prime} \mathrm{N}$, $37^{\circ} 34^{\prime} \mathrm{E}, 518 \mathrm{~m}, 20.04 .2010 ; 3 \delta^{\top} \sigma^{\top}, 2$ 우, İslahiye, Fevzipaşa, $37^{\circ} 04^{\prime} \mathrm{N}, 37^{\circ} 36 \mathrm{E}, 513 \mathrm{~m}, 10.05 .2011$; Oğuzeli, $37^{\circ} 57^{\prime} \mathrm{N}, 37^{\circ} 30^{\prime} \mathrm{E}, 716 \mathrm{~m}, 28.6 .2019$; Det: Yaran M. (Leg. Yaran, Görmez and Koyuncu).

Goniurellia longicauda Freidberg, 1980

Material examined: $1 \delta^{\top}$, Karkamış, $36^{\circ} 49^{\prime} \mathrm{N}, 37^{\circ} 45^{\prime} \mathrm{E}$, 507 m, 03.06.2010; Det: Yaran M. (Leg. Yaran, Görmez and Koyuncu)

Goniurellia tridens (Hendel, 1910) Figure 1

Material examined: 1 $\hat{\sigma}, 1$ q, Gaziantep, İslahiye, Fevzipaşa, $37^{\circ} 15^{\prime} \mathrm{N}, 37^{\circ} 37^{\prime} \mathrm{E}, 523 \mathrm{~m}, 10.05 .2021$; Det: Yaran M. (Leg. Yaran, Görmez and Koyuncu).

Comment: Goniurellia tridens differs from other Goniurellia Hendel 1927 species with following characters; cell $\mathrm{R}_{4+5}$ usually with 1-3 hyaline dots; distance between crossveins usually 1.5-2 times a s long as $\mathrm{r}-\mathrm{m}$ crossvein; 2 brown rays present in cell $\mathrm{D}$ in addition to ray on dm-cu ray usually reaching middle of the cell (For detailed description see Freidberg and Kugler, 1989). $G$. tridens distributed in Turkmenia, Bukhara, Iran, Pakistan, India, Israel and Saudi Arabia (Freidberg and Kugler, 1989). This species recorded for the first time together this research in Turkey. 
Table 1. Current list of fruit fly fauna in Gaziantep province

\begin{tabular}{|c|c|c|}
\hline No & Species & Reference \\
\hline 1. & Acanthiophilus helianthi (Rossi, 1794) & Yaran, 2009 \\
\hline 2. & Aciura coryli (Rossi, 1790) & Yaran, 2009 \\
\hline 3. & Actinoptera discoidea (Fallen, 1814)* & Current study \\
\hline 4. & Bactrocera oleae (Rossi, 1790)* & Current study \\
\hline 5. & Campiglossa producta (Loew, 1844) & Yaran, 2009 \\
\hline 6. & Ceratitis capitata (Wiedemann, 1824) & Yaran et al., 2018b \\
\hline 7. & Chaetorellia carthami Stackelberg, 1929 & Yaran, 2009 \\
\hline 8. & Chaetorellia jaceae (Robineau - Desvoidy, 1830)* & Current study \\
\hline 9. & Chaetorellia loricata (Rondani, 1830) & Yaran, 2009 \\
\hline 10. & Chaetorellia succinea (Costa, 1844) & Yaran, 2009 \\
\hline 11. & Chaetostomella cylindrica (Robineau - Desvoidy, 1830) & Yaran, 2009 \\
\hline 12. & Euaresta bullans (Wiedemann, 1830) & Yaran, 2009 \\
\hline 13. & Euleia heraclei (Linnaeus, 1758)* & Current study \\
\hline 14. & Goniurellia longicauda Freidberg, 1980* & Current study \\
\hline 15. & Goniurellia tridens (Hendel, 1910)* & Current study \\
\hline 16. & Rhagoletis cerasi (Linnaeus, 1758)* & Current study \\
\hline 17. & Sphenella marginata (Fallen, 1814) & Yaran, 2009 \\
\hline 18. & Tephritis acanthiophilopsis Hering, 1938* & Current study \\
\hline 19. & Tephritis dioscurea (Loew, 1856) & Yaran, 2009 \\
\hline 20. & Tephritis hurvitzi Freidberg, 1981 & Yaran, 2009 \\
\hline 21. & Tephritis merzi Freidberg and Kütük, 2002* & Current study \\
\hline 22. & Tephritis postica (Loew, 1844) & Yaran, 2009 \\
\hline 23. & Tephritis praecox (Loew, 1844)* & Current study \\
\hline 24. & Tephritomyia lauta (Loew, 1869) & Yaran, 2009 \\
\hline 25. & Terellia gynaecochroma (Hering, 1937) & Yaran, 2009 \\
\hline 26. & Terellia luteola (Wiedemann, 1830) & Yaran, 2009 \\
\hline 27. & Terellia serratulae (Linnaeus, 1758) & Yaran, 2009 \\
\hline 28. & Terellia virens (Loew, 1846) & Yaran, 2009 \\
\hline 29. & Terellia winthemi (Meigen, 1826)* & Current study \\
\hline 30. & Trupanea amoena (Frauenfeld, 1857) & Yaran, 2009 \\
\hline 31. & Trupanea stellata (Fuesslin, 1775) & Yaran, 2009 \\
\hline 32. & Urophora affinis (Frauenfeld, 1857) & Yaran, 2009 \\
\hline 33. & Urophora aprica (Fallen, 1814)* & Current study \\
\hline 34. & Urophora congrua Loew, $1862 *$ & Current study \\
\hline 35. & Urophora cuspidata (Meigen, 1826) & Yaran, 2009 \\
\hline 36. & Urophora dzieduszyckii Frauenfeld, 1867 & Yaran, 2009 \\
\hline 37. & Urophora jaceana (Hering, 1935) & Yaran, 2009 \\
\hline 38. & Urophora mauritanica Macquart, 1851 & Yaran, 2009 \\
\hline 39. & Urophora phalolepidis Merz and White, 1991* & Current study \\
\hline 40. & Urophora quadrifasciata (Meigen, 1826) & Yaran, 2009 \\
\hline 41. & Urophora solstitialis (Linnaeus, 1758) & Yaran, 2009 \\
\hline 42. & Urophora stylata (Fabricius, 1775) & Yaran, 2009 \\
\hline 43. & Urophora tenuior Hendel, 1910 & Yaran, 2009 \\
\hline 44. & Urophora terebrans (Loew, 1850)* & Current study \\
\hline
\end{tabular}

Rhagoletis cerasi (Linnaeus, 1758)

Material examined: 2 ๙ิ $\widehat{\partial}, 1$ q, Gaziantep, Şehitkamil, Bilek, $37^{\circ} 12^{\prime} \mathrm{N}, 37^{\circ} 56 \mathrm{E}, 513$ m, 10.07.2018; Det: Yaran M. (Leg. Yaran, Görmez and Koyuncu).

Tephritis acanthiophilopsis Hering, 1938

Material examined: $1 \hat{\partial}$, Gaziantep, Araban, Suvarlı, $37^{\circ} 33^{\prime} \mathrm{N}, 37^{\circ} 38^{\prime} \mathrm{E}, 968 \mathrm{~m}, 16.06 .2010$; Det: Yaran M. (Leg. Yaran, Görmez and Koyuncu).

Tephritis merzi Freidberg and Kütük, 2002

Material examined: $1 \hat{\jmath}$, Gaziantep, Şahinbey, Türközü, 36 $05^{\prime} \mathrm{N}, 37^{\circ} 21^{\prime} \mathrm{E}, 691 \mathrm{~m}, 10.05 .2010$; Det: Yaran M. (Leg. Yaran, Görmez and Koyuncu).
Tephritis praecox (Loew, 1844)

Material examined: 1 ๙े, 1 q, Gaziantep, Şehitkamil, Bilek, $37^{\circ} 12^{\prime} \mathrm{N}, 37^{\circ} 56 \mathrm{E}, 513$ m, 10.07.2018; Det: Yaran M. (Leg. Yaran, Görmez and Koyuncu).

Terellia winthemi (Meigen, 1826)

Material examined: 1 त, Gaziantep, Araban, Suvarl1, $37^{\circ} 33^{\prime} \mathrm{N}, 37^{\circ} 38^{\prime} \mathrm{E}, 968 \mathrm{~m}, 16.06 .2010$; Det: Yaran M. (Leg. Yaran, Görmez and Koyuncu).

Urophora aprica (Fallen, 1814)

Material examined: 1 +, 1 , Gaziantep, Şahinbey, Cevizli, $36^{\circ} 54^{\prime} \mathrm{N}, 37^{\circ} 03^{\prime} \mathrm{E}, 628 \mathrm{~m}, 01.06 .2010 ; 1$ ô, Karkamıs, $36^{\circ} 49^{\prime}$ N, $37^{\circ} 45^{\prime}$ E, 507 m, 03.06.2010; Det: Yaran M. (Leg. Yaran, Görmez and Koyuncu). 

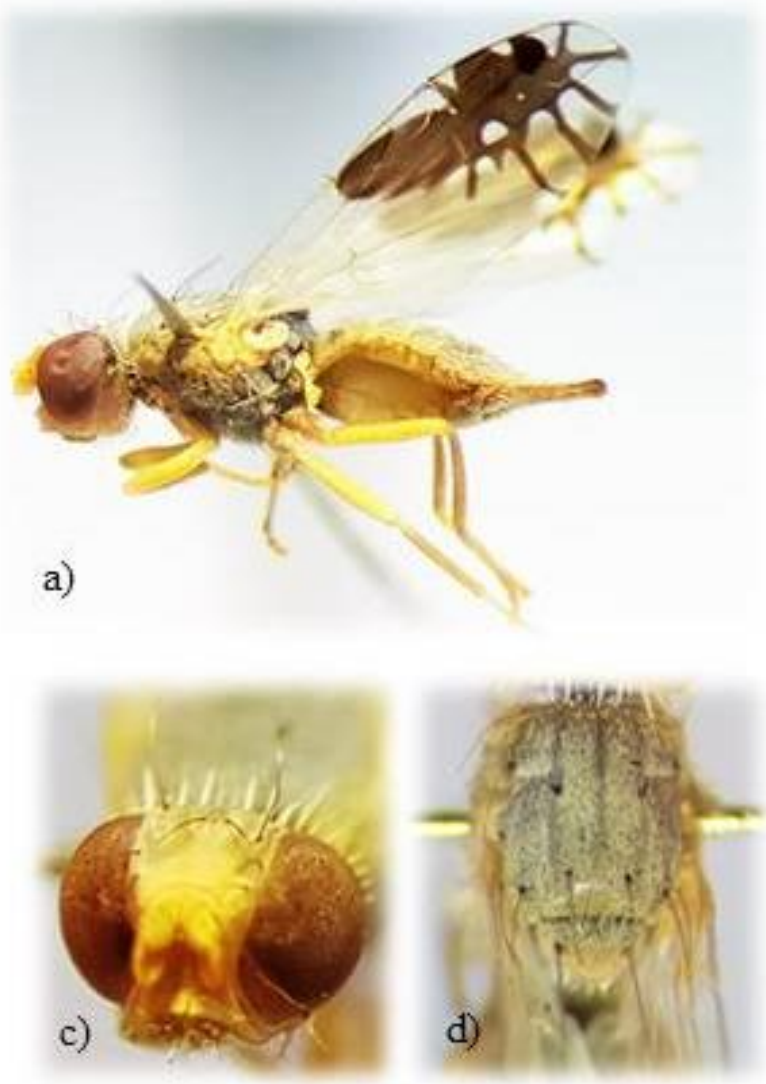
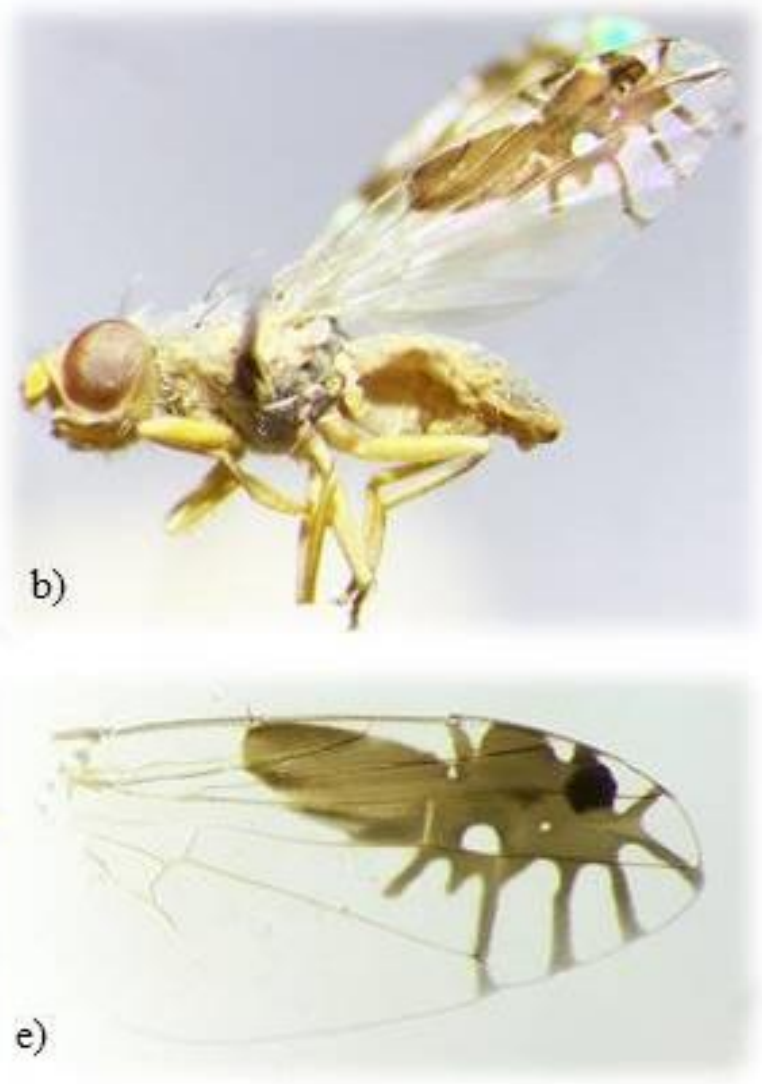

Figure 1. Figures of Goniurellia tridens a) female, b) male, c) head, d) thorax, e) wing

\section{Urophora congrua Loew, 1862}

Material examined: 1 స, 2 우, Gaziantep, Şahinbey, Kürüm, $36^{\circ} 57^{\prime} \mathrm{N}, 37^{\circ} 09^{\prime} \mathrm{E}, 903 \mathrm{~m}, 17.06 .2010$; Det: Yaran M. (Leg. Yaran, Görmez and Koyuncu).

\section{Urophora phalolepidis Merz and White, 1991}

Material examined: $1 \delta^{\lambda}, 1$ ㅇ, Gaziantep, Şahinbey, Türközü, $36^{\circ} 5^{\prime} \mathrm{N}, 37^{\circ} 21^{\prime} \mathrm{E}, 691 \mathrm{~m}, 10.05 .2010 ; 1$ क, İslahiye, Türkbahçe, $37^{\circ} 04^{\prime} \mathrm{N}, 36^{\circ} 37^{\prime} \mathrm{E}, 519 \mathrm{~m}$, 10.05.2010; 1 ㅇ, Karkamış, $36^{\circ} 49^{\prime} \mathrm{N}, 37^{\circ} 45^{\prime} \mathrm{E}, 507 \mathrm{~m}$, 03.06.2010; $1 \mathrm{\delta}^{\lambda}$, Araban, Beydili, $37^{\circ} 30^{\prime} \mathrm{N}, 37^{\circ} 39^{\prime} \mathrm{E}, 910$

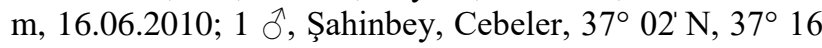
E, 946 m, 17.06.2010; Det: Yaran M. (Leg. Yaran, Görmez and Koyuncu).

Urophora terebrans (Loew, 1850)

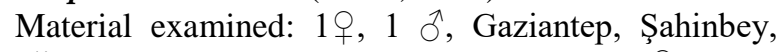
Töreli, $36^{\circ} 53^{\prime} \mathrm{N}, 37^{\circ} 21^{\prime} \mathrm{E}, 670 \mathrm{~m}, 20.04 .2010 ; 1$ ㅇ, Nizip, $36^{\circ} 59^{\prime} \mathrm{N}, 37^{\circ} 40^{\prime} \mathrm{E}, 607 \mathrm{~m}, 23.04 .2010$; 1 엉 İslahiye, Türkbahçe, $37^{\circ} 04^{\prime} \mathrm{N}, 36^{\circ} 37^{\prime} \mathrm{E}, 519 \mathrm{~m}, 10.05 .2010 ; 1$ 'े, Şahinbey, Türközü, $36^{\circ} \quad 05^{\prime} \mathrm{N}, 37^{\circ} 21^{\prime} \mathrm{E}, 691 \mathrm{~m}$, 10.05.2010; 1 ㅇ, Şahinbey, Akpınar, $37^{\circ} 45^{\prime} \mathrm{N}, 37^{\circ} 44^{\prime} \mathrm{E}$, $825 \mathrm{~m}, 15.05 .2010 ; 1$ ô, Şahinbey, Cevizli, $36^{\circ} 54^{\prime} \mathrm{N}, 37^{\circ}$ $03^{\prime} \mathrm{E}, 628 \mathrm{~m}, 01.06 .2010$; 1 ㅇ, Karkamış, $36^{\circ} 49^{\prime} \mathrm{N}, 37^{\circ} 45^{\circ}$ E, $507 \mathrm{~m}, 03.06 .2010 ; 1$ 은, Yavuzeli, $37^{\circ} 19^{\prime} \mathrm{N}, 37^{\circ} 40^{\prime} \mathrm{E}$,

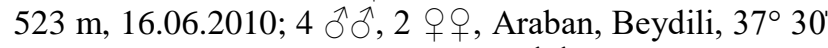

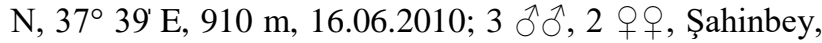
Uğurtepe, $36^{\circ} 57^{\prime} \mathrm{N}, 37^{\circ} 05^{\prime} \mathrm{E}, 774 \mathrm{~m}, 17.06 .2010 ; 1$ ๙े, 2 우오, Şahinbey, Kürüm, $36^{\circ} 57^{\prime} \mathrm{N}, 37^{\circ} 09^{\prime} \mathrm{E}, 903 \mathrm{~m}$, 17.06.2010; 3 ○े $^{\Uparrow}, 1$ 우우, İslahiye, Fevzipaşa, $37^{\circ} 06 \mathrm{~N}$, $36^{\circ} 36 \mathrm{E}, 1040 \mathrm{~m}$; 14.07.2010; Det: Yaran M. (Leg. Yaran, Görmez and Koyuncu).

\section{Acknowledgement}

We thank the Scientific and Technological Research Council of Turkey (TÜBİTAK, Project Number 108T465). We also thank to Prof. Dr. Murat Kütük for all support during preparing this manuscript.

\section{References}

Bayrak N. 2011. Kuzeydoğu Anadolu Bölgesi Tephritinae ve Terellinae (Diptera: Tephritidae) Türleri Üzerinde Faunistik ve Sistematik Çalışmalar. $\mathrm{PhD}$ Dissertation, Atatürk University, Institute of Sciences, Erzurum, Turkey.

Çalışkan Keçe AF, Çatal BÖ, Ulusoy MR. 2019. A new invasive species in Turkey: Dacus ciliatus Loew, 1862 (Diptera: Tephritidae). Turkish Journal of Entomology, 43(1): 25-30.

Freidberg A, Kugler J. 1989. Fauna Palaestina Insecta IV. Diptera: Tephritidae. Israel Academy of Sciences and Humanities.

Freidberg A. 2016. New taxa of Carpomyini, with special emphasis on Goniglossum (Diptera: Tephritidae: Trypetinae). Zootaxa, 4144(1): 054-070.

Görmez V, Kütük M. 2020. Fruit fly (Diptera: Tephritidae) fauna of Çorum and Sinop provinces with two new records for Turkey. Turkish Journal of Entomology 44(1): 23-38.

Görmez V. 2011. Kahramanmaraş İlinde Meyve Sinekleri (Diptera: Tephritidae) Faunası ve Sistematiği Üzerine Araştırmalar. MSc Dissertation, Gaziantep University, Institute of Sciences, Gaziantep, Turkey.

Hendel F. 1927. 49. Trypetidae die Fliegen der Palaerktischen Region. Book on Demand. ASIN: B0068PDJF4.

Korneyev VA, Kolcsar LP. 2015. First records of Trypetini (Diptera: Tephritidae: Trypetinae) from Europe. Ukrainska Entomofaunistyka, 6 (3): 34. 
Korneyev VA, Evstigneev DA, Karimpour Y, Kütük M, Mohamadzade Namin S, Koyuncu MÖ, Yaran M. 2013. Revision of the Terellia virens group (Diptera, Tephritidae) with description of three new species. Vestnik Zoologii, 47 (1): 3-25.

Korneyev VA, White IM. 1993. Fruit-flies of the Eastern Palaearctic species of Urophora R.-D. (Diptera: Tephritidae). II. Review of species of the subgenus Urophora s. str. (Communication 2). Entomologicheskoe obozrenie, 72(1): 232-247.

Korneyev VA, White IM. 1999. Fruit flies of genus Urophora RD. (Diptera: Tephritidae) of east palaerctic. III. key to species. Entomologicheskoe Obozrenie, 78(2): $464-482$.

Korneyev VA. 2003. New and Little-Known Tephritidae (Diptera, Cyclorrhapha) from Europe. Vestnik Zoologii. 37(3): 3-12.

Korneyev VA. 2006. A revision of the quadratula group of the genus Terellia Robineau-Desvoidy (Diptera: Tephritidae). In: Freidberg A., ed. Biotaxonomy of Tephritoidea: Israel Journal of Zoology, 35-36: 341-366.

Kütük M, Bayrak N, Hayat R. 2011. A new species of Terellia (Diptera: Tephritidae) from Turkey with a key to Turkish species of the subgenus Cerajocera. Turkish Journal of Entomology, 35 (2): 207-213.

Kütük M, Bayrak N, Hayat R. 2012. A new species of Tephritis Latreille (Diptera: Tephritidae) from Turkey. Turkish Journal of Zoology, 36(4): 475-480.

Kütük M, Yaran M, Hayat R, Koyuncu MÖ, Görmez V, Aytekin HU. 2013. The determination of fruit fly (Diptera: Tephritidae) fauna in Adıyaman, Kilis, and Şanlıurfa provinces with a new record for Turkish fauna. Turkish Journal of Zoology, 37: 38-49.

Kütük M, Yaran M. 2020. A new species and a new record of Tephritis Latreille, 1804 (Diptera: Tephritidae) from Turkey. Turkish Journal of Entomology, 22(2): 215-222.

Kütük M. 2003a. Güneybatı Anadolu Bölgesi Tephritidae Faunası ve Sistematiği Üzerine Araştırmalar. PhD Dissertation, Çukurova University, Institute of Sciences, Adana, Turkey.

Kütük M. 2003b. Doğu Akdeniz Bölgesi Urophora RobineauDesvoidy (Diptera: Tephritidae) faunası ve sistematiği üzerine araştırmalar. Turkish Journal of Entomology, 27(2): 149-160.

Kütük M. 2006. The Fauna and Systematics of the Genus Tephritis Latreille, 1804 (Diptera: Tephritidae) with a Key to the Species of Tephritis in Turkey. Turkish Journal of Zoology, 30: 345-356
Merz B. 1994. Diptera: Tephritidae. Insecta Helvetica Fauna. HGE Press, Geneva, 198 pp.

Namin SM, Korneyev S.V, 2015. Revision of Heringina Aczél, 1940 (Diptera: Tephritidae), with description of a new species from Iran and Turkey. Zootaxa 3949 (1): 111-122.

Pape T, Vladimir B, Mikhail BM. 2011. Order Diptera Linnaeus, 1758. In: Zhang, Z. - Q. (Ed.) Animal biodiversity: An outline of higher - level classification and survey of taxonomic richness. Zootaxa, 3148: 222-229.

Sarwar M. 2015. Quarantine treatments for mortality of eggs and larvae of fruit flies (Diptera: Tephritidae) invading fresh horticulture Perishable Produces. Int. J. Anim. Biol. 1, 196201.

White IM. 1988. Tephritid flies (Diptera: Tephritidae). Handbook for the identification of British insects. Intl Specialized Book Services. ISBN-10: 0901546682.

Yaran M, Görmez V. 2020. A new species of Tephritis Latreille, 1804 (Diptera: Tephritidae) from Turkey. Zootaxa 4838(2):283-288

Yaran M, Kütük M, Görmez V, Koyuncu MÖ. 2018a. A new species and additional record of Terellia Robineau - Desvoidy (Diptera: Tephritidae) from Turkey with a key for the Cerajocera group. Turkish Journal of Zoology, 42: 661-665.

Yaran M, Kütük M, Görmez V, Koyuncu MÖ. 2018b. Some additional notes on fruit fly (Diptera: Tephritidae) fauna and a new genera and species record from Turkey. Biological Diversity and Conservation, 11(3): 141-144.

Yaran M, Kütük M. 2012. The fruit flies (Diptera: Tephritidae) fauna of Gaziantep province, Turkey. Munis Entomology and Zoology, 7 (2): 957-969.

Yaran M, Kütük M. 2014. A new species of Urophora Robineau - Desvoidy, 1830 (Diptera: Tephritidae) from Turkey with a key to the dzieduszyckii group. Turkish Journal of Entomology, 38: 149-155.

Yaran M, Kütük M. 2015. Two new records of fruit flies (Diptera: Tephritidae) with fauna of Aksaray and Mersin provinces. Turkish Journal of Zoology, 39: 1056-1070.

Yaran M, Kütük M. 2016. Fruit flies (Diptera: Tephritidae) fauna in Nevşehir and Niğde provinces with a new record from Turkey. Turkish Journal of Zoology, 40(5): 785-800.

Yaran M. 2014. Aksaray, Mersin, Nevşehir ve Niğde illerinde meyve sinekleri (Diptera: Tephritidae) faunası ve sistematiği üzerine araştırmalar. $\mathrm{PhD}$ Thesis, Gaziantep University, Institute of Sciences, Gaziantep, Turkey. 